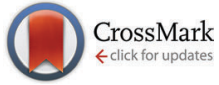

Cite this: J. Mater. Chem. C, 2016 , 4, 3905

Received 20th November 2015, Accepted 12th February 2016

DOI: $10.1039 / c 5 t c 03891 j$

www.rsc.org/MaterialsC

\title{
Transition-metal-nitride-based thin films as novel energy harvesting materials
}

\author{
Per Eklund, ${ }^{* a}$ Sit Kerdsongpanya ${ }^{a}$ and Björn Alling ${ }^{\text {ab }}$
}

\begin{abstract}
The last few years have seen a rise in the interest in early transition-metal and rare-earth nitrides, primarily based on $\mathrm{ScN}$ and $\mathrm{CrN}$, for energy harvesting by thermoelectricity and piezoelectricity. This is because of a number of important advances, among those the discoveries of exceptionally high piezoelectric coupling coefficient in ( $\mathrm{Sc}, \mathrm{Al}) \mathrm{N}$ alloys and of high thermoelectric power factors of $\mathrm{ScN}$ based and $\mathrm{CrN}$-based thin films. These materials also constitute well-defined model systems for investigating thermodynamics of mixing for alloying and nanostructural design for optimization of phase stability and band structure. These features have implications for and can be used for tailoring of thermoelectric and piezoelectric properties. In this highlight article, we review the ScN- and $\mathrm{CrN}$-based transition-metal nitrides for thermoelectrics, and drawing parallels with piezoelectricity. We further discuss these materials as a models systems for general strategies for tailoring of thermoelectric properties by integrated theoretical-experimental approaches.
\end{abstract}

\section{Introduction}

In the last few years, the early transition-metal and rare-earth nitrides, primarily based on $\mathrm{ScN}$ and $\mathrm{CrN}$, have emerged as an unexpected class of materials for energy harvesting by thermoelectricity and piezoelectricity and more generally for conversion of heat or mechanical energy to electricity. Largely ignored for these purposes until around 2010, this class of materials is now on the rise because of a number of fundamental advances, among those the discoveries of exceptionally high piezoelectric coupling coefficient in $(\mathrm{Sc}, \mathrm{Al}) \mathrm{N}$ alloys ${ }^{1}$ and of remarkably high thermoelectric power factors of ScN-based ${ }^{2,3}$ and CrN-based ${ }^{4,5}$ thin films. These materials also constitute well-defined model systems for investigating thermodynamics of mixing for alloying and nanostructural design for optimization of phase stability and band structure. These features have implications for and can be used for tailoring of thermoelectric and piezoelectric properties.

The process of energy harvesting is the capture of energy from ambient sources and storage and/or application for use as power sources. There is a wide range of ambient sources, including solar, wind, electromagnetic radiation, mechanical (kinetic) energy, and thermal energy. Energy harvesting differs conceptually from, e.g., oil and coal power, fuel cells, or batteries, that involve active combustion of a fuel or conversion of stored chemical energy to electricity. Furthermore, the term energy

\footnotetext{
${ }^{a}$ Thin Film Physics Division, Linköping University, IFM, 58183 Linköping, Sweden. E-mail:perek@ifm.liu.se

${ }^{b}$ Max-Planck-Institut für Eisenforschung GmbH, D-40237 Düsseldorf, Germany
}

harvesting is typically reserved for capturing energy for powering small, low-power devices, usually off-grid or otherwise autonomous. The term does not include, e.g., solar-power and wind-power plants, although the fundamental concept is the same.

In this highlight article, we review the $\mathrm{ScN}$ - and $\mathrm{CrN}$-based transition-metal nitrides for energy-harvesting applications by thermoelectrics (harvesting of ambient heat), drawing parallels with piezoelectricity (harvesting of mechanical vibrations). It is further intended as an example of general strategies for tailoring of thermoelectric properties by integrated theoretical-experimental approaches.

\section{Brief introduction to thermoelectricity}

Thermoelectric devices harvest thermal energy (temperature gradients) into electricity, and can also be used for environmentally friendly refrigeration, without moving parts or malign liquids or gases. Most other conversion systems (such as power plants) become less efficient as they are scaled down in size and power, but thermoelectrics benefit from low- to medium-power and -size application. Thus, potential contributions of thermoelectrics are in applications with relatively low power levels used in large numbers (for example in personal computers, automotive applications, and consumer electronics). The efficiency of a thermoelectric material at a temperature $T$ is related to the dimensionless figure of merit $Z T$, where $Z=S^{2} \sigma / \kappa$. Here, $\sigma$ and $\kappa$ are the electrical and thermal conductivities, respectively, and $S$ is the Seebeck coefficient $\Delta V / \Delta T$, i.e., the voltage in response 
to a temperature gradient. The thermal conductivity is given by $\kappa=\kappa_{1}+\kappa_{\mathrm{e}}$, where the subscripts 1 and e denote the lattice (phonon) and electron contributions, respectively. Thus, a high $Z T$ requires a good electrical conductor with high Seebeck coefficient but low thermal conductivity. ${ }^{6}$ For traditional thermoelectric materials like tellurides and antimonides, $Z T \approx 1$ at room temperature. At first glance, it seems easy to increase $Z T$ by, e.g. , increasing the conductivity by a factor of $2-4$. However, basic transport theory in solids implies that $S, \sigma$, and $\kappa$ are interrelated; increasing $\sigma$ by increasing the charge carrier concentration results in lower $S$ and increased $\kappa$, yielding no improvement in $Z T$.

The delicate interdependence of the three parameters $S, \sigma$, and $\kappa$ requires novel approaches to advance the field of thermoelectrics, which has led to extensive efforts on nanostructural design. ${ }^{7,8}$ Predictions in the mid-1990s suggested that $Z T$ could

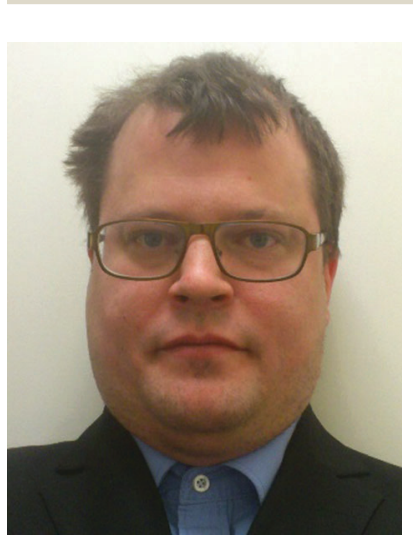

Per Eklund

Per Eklund (PhD 2007) is Associate Professor and leads the Energy Materials Group of the Thin Film Physics Division at Linköping University, Sweden, where he joined the faculty in 2009. His main research interests encompass the wider area of thin-film ceramics primarily for energy applications, including thermoelectrics, ionic conductors, inherently nanolaminated 'MAX phases' and their 2D counterparts 'MXenes', and for electrical contacts and tools. Eklund has $\sim 100$ published articles and an $h$ index of 24. He is an ERC Starting Grant Holder, Wallenberg Academy Fellow, member of the Young Academy of Sweden (2011-2016), and Editor of the journal Vacuum.

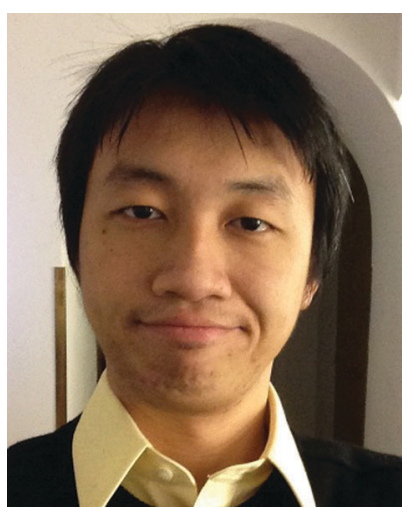

Sit Kerdsongpanya
Sit Kerdsongpanya received his BSc degree from Chulalongkorn University, Bangkok, Thailand, in 2008 and his MSc degree in the International Master's Programme at Linköping University in 2010. He graduated with a PhD from Linköping University in 2015 on the development and design of thermoelectric thin films of epitaxial ScN and ScN-based alloys. He is currently a postdoctoral researcher at Linköping University. be enhanced by quantum confinement. ${ }^{9}$ In 2001 , thermoelectric superlattice devices of $\mathrm{Bi}_{2} \mathrm{Te}_{3} / \mathrm{Sb}_{2} \mathrm{Te}_{3}$ with remarkably high $Z T$ $(\sim 2.4)$ caused a surge of interest. ${ }^{10}$ Materials with such high $Z T$, however, are restricted to laboratory devices, which has prevented their success in practice. ${ }^{11}$ At present, the main achievement of nanostructuring is reduction of the lattice thermal conductivity $^{12,13}$ rather than improvements due to quantum confinement of charge carriers. ${ }^{14,15}$

There is therefore a need to introduce mechanisms that, in addition to reducing $\kappa$, also enhance the thermoelectric power factor $\left(S^{2} \sigma\right)$. One innovative approach is to consider theoretically what band structure a hypothetical material should have to maximize $Z T$. Mahan and Sofo ${ }^{16}$ predicted this in the 1990s, and others have more recently refined the picture. ${ }^{17}$ For a given $\kappa_{1}$, the ideal transport-distribution function that maximizes $Z T$ is a bounded delta function, approximately realized in practice as a sharp function with a large slope in the density of states (DOS) at the Fermi level $E_{\mathrm{F}}{ }^{9,15}$ These approaches are the base for modern strategies for the development of thermoelectrics: band-structure optimization to emulate the ideal band structure, combined with nanostructural design to reduce the thermal conductivity.

\section{The early transition-metal nitrides}

\section{A. Overall trends}

The early transition-metal nitrides - and their alloys - based on group-4 (Ti, Zr, and Hf) or group-5 (V, Nb, and Ta) metals, are long-established in applications as hard, wear-resistant coatings, with TiN being the archetype. While they are hard and exhibit other typical ceramic properties, these nitrides are metallic in nature with respect to electrical properties and in fact very good conductors, with typical resistivities in the approximate range 10-30 $\mu \Omega \mathrm{cm}$ (in comparison, noble metals have resistivities of a few $\mu \Omega \mathrm{cm}$, and Ti metal about $40 \mu \Omega \mathrm{cm}) .{ }^{18}$ Thus, they find extensive use as conducting permanent contact layers and diffusion barriers in microelectronics.

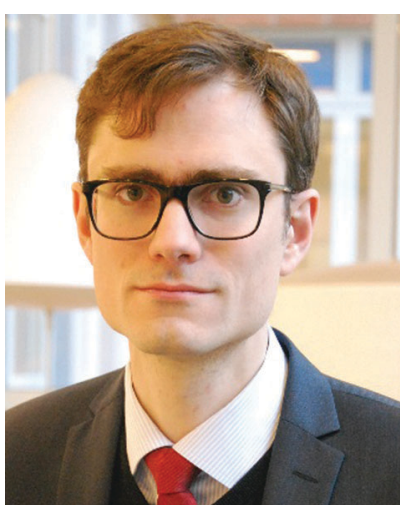

Björn Alling
Björn Alling is currently (20152018) a visiting senior researcher at the Max-Planck-Institute for Iron Research (MPIE) in Düsseldorf, Germany. In parallel, he holds a permanent position as associate professor in Linköping. He has two PhD degrees, from EPFL in Lausanne, Switzerland (2009) and from Linköping University (2010). He has published $\sim 60$ scientific papers and holds a Swedish Research Council International Career Grant. He has made important contributions to modeling of the complex material CrN and its alloys, method development for treatment of magnetic materials at high temperature, and alloy theory for multicomponent alloys. 
Reducing the valency by one from Ti or increasing it by one from $\mathrm{V}$, i.e., moving to groups 3 or 6 in the periodic table, results in drastically altered properties. The cubic rock-saltstructured $\mathrm{ScN}$ and $\mathrm{CrN}$ are both narrow-bandgap semiconductors. Sc has three valence electrons that together with the three $2 p$ valence electrons of $\mathrm{N}$ complete the filling of the bonding states formed by nearest neighbor hybridization of mainly $\mathrm{N} 2 \mathrm{p}$ and Sc $3 d e_{g}$ with some $\mathrm{Sc} 4 \mathrm{~s}$ character. The $3 \mathrm{~d} \mathrm{t}_{2 \mathrm{~g}}$-orbitals, on the other hand, are completely empty, in contrast to the group-4 and -5 transition metals, and the Fermi level drops below the conduction band edge, causing $\mathrm{ScN}$ to become semiconducting. In $\mathrm{CrN}$, with three more electrons than $\mathrm{ScN}$, the non-bonding $\mathrm{Cr}$ $3 \mathrm{~d} \mathrm{t}_{2 \mathrm{~g}}$-band is half-filled. This causes a spin splitting of the band which give $\mathrm{Cr}$ atoms of $\mathrm{CrN}$ a distinct local magnetic moment approaching $3 \mu_{\mathrm{B}}{ }^{19}$ As a consequence, also in this material, the Fermi level falls into a bandgap, this time between occupied spin-up non-bonding $\mathrm{Cr} 3 \mathrm{~d} \mathrm{t}_{2 \mathrm{~g}}$ and an unoccupied mixture of mostly anti-bonding spin-up $\operatorname{Cr} 3 \mathrm{~d}_{\mathrm{g}}$, and non-bonding spindown $\operatorname{Cr} 3 \mathrm{~d} \mathrm{t}_{2 \mathrm{~g} \cdot}{ }^{20,21}$

The effect on resistivity is illustrated in Fig. 1, from an early study by Gall et al. ${ }^{22}$ who investigated $\mathrm{Ti}_{x} \mathrm{Sc}_{1-x} \mathrm{~N}$ epitaxial thin films. Pure TiN is a good conductor and exhibits a typical roomtemperature resistivity value around $20 \mu \Omega \mathrm{cm}$, and the archetypical metallic temperature dependence with constant resistivity at low temperature dominated by scattering from vacancies, defects, and impurities. As temperature increases, the resistivity increases linearly with the scattering dominated by electronphonon coupling. For low Sc content in the $\mathrm{Ti}_{x} \mathrm{Sc}_{1-x} \mathrm{~N}$ alloy, this behavior is initially retained, but for higher Sc content, an increase in resistivity is observed at cryogenic temperature and for pure $\mathrm{ScN}$ this effect is dominant indicating semiconducting behavior. It needs to be stressed, though, that this is highly dependent on impurities and dopants.

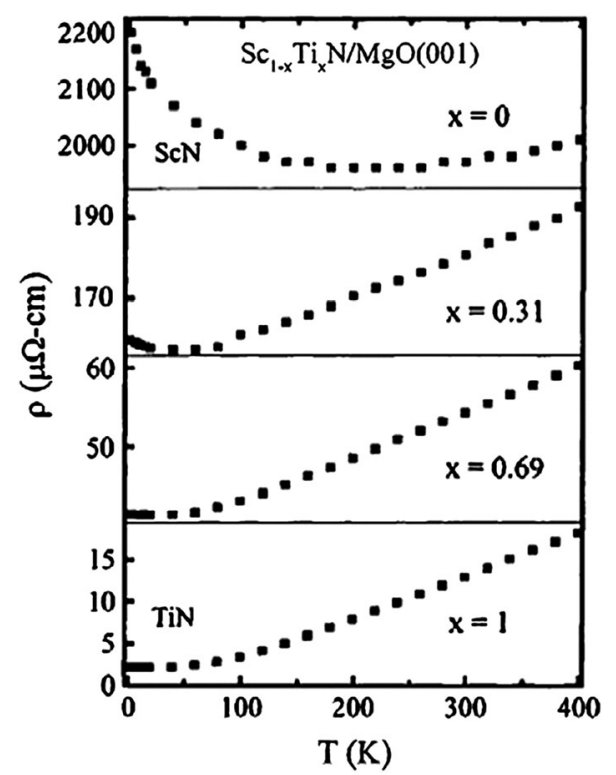

Fig. 1 Electrical properties of ScN, TiN, and ScTiN alloys. From Gall et al. ${ }^{22}$ (Copyright American Institute of Physics, used with permission).

\section{B. $\mathrm{ScN}$}

ScN, like the group-4 and group-5 transition-metal nitrides, is an interstitial (cubic NaCl structure) nitride following Hägg's rule; that interstitial nitrides and carbides are formed when the radii ratio between the nonmetal and metal atoms is smaller than $0.59 .^{23} \mathrm{ScN}$ has similar hardness as the other transition-metal nitrides, $21 \mathrm{GPa}$, and is stable at high temperature (melting point $2550^{\circ} \mathrm{C}$ ), while it is prone to oxidation if used in air above $600{ }^{\circ} \mathrm{C} .{ }^{24-26}$

As stated above, $\mathrm{ScN}$ is a narrow-bandgap semiconductor. The fundamental bandgap is $\sim 0.9 \mathrm{eV}$ and the direct (optical bandgap) is $\sim 2.1 \mathrm{eV}$. This was, however, a topic of debate for a rather long time with numerous studies yielding conflicting results as to whether $\mathrm{ScN}$ was a semiconductor, a semimetal or even a metal. ${ }^{27-30}$ The reason for these discrepancies are that it is challenging to produce pure $\mathrm{ScN}$. Sc has a high affinity to oxygen $^{31}$ and, if not synthesized in a pure ultrahigh-vacuum environment, can readily contain large amounts of oxygen impurities, as well as contaminations from residual hydrocarbons. Free carriers from impurities can result in large inaccuracies in determinations of optical bandgaps. ${ }^{30}$ Furthermore, processing of scandium ore involves a purification step with fluoride reduction, ${ }^{32}$ which results in scandium raw materials often containing fluorine impurities.

There are relatively many studies on thin-film growth of $\mathrm{ScN}$. Among the methods used, magnetron sputter deposition, 2,22,30,33-35 chemical vapor deposition $^{24,36}$ and molecular beam epitaxy ${ }^{37-42}$ are the most common. Irrespective of method, the aspects of reactivity and oxygen and/or fluorine uptake (or other impurities) are essential in thin-film growth of $\mathrm{ScN}$, stressing the need for a pure environment.

For a transition-metal nitride, $\mathrm{ScN}$ exhibit an anomalously high thermoelectric power factor ${ }^{2,3} S^{2} \sigma . S^{2} \sigma$ is in the range 2.5-3.3 $\mathrm{W} \mathrm{m}^{-1} \mathrm{~K}^{-2}$, well on par with established thermoelectric materials such as PbTe. ${ }^{43}$ This is illustrated in Fig. 2, where ScN (our data from ref. 2 and the results of Burmistrova et al. ${ }^{3}$ ) are shown in relation to a typical value for n-type PbTe. In comparison, the power factor of $\mathrm{Bi}_{2} \mathrm{Te}_{3}$ is somewhat higher at above $4 \mathrm{~W} \mathrm{~m}^{-1} \mathrm{~K}^{-2}$. The thermal conductivity of $\mathrm{ScN}$, though, is much higher than for these tellurides, in the range 8-12 $\mathrm{W} \mathrm{m}^{-1} \mathrm{~K}^{-1,3,24,44}$ and would need to be drastically reduced to enable application of

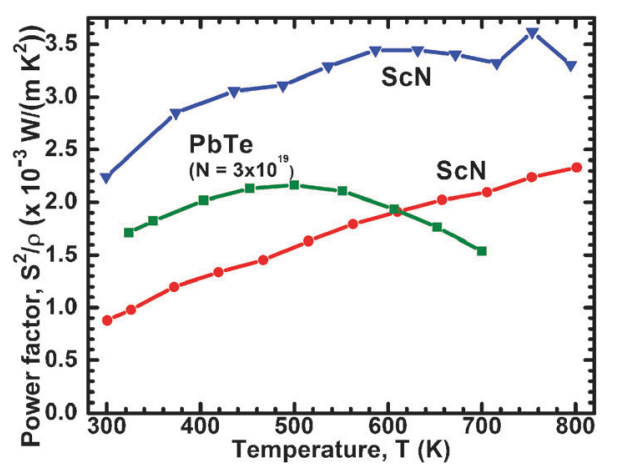

Fig. 2 Thermoelectric power factor $S^{2} \sigma$ of $\operatorname{ScN}$ (the bottom curve shows our first data from ref. 2 and the top curve are the data of Burmistrova et al.). ${ }^{3}$ The example data for PbTe are adapted from Sootsman et al. ${ }^{43}$ 


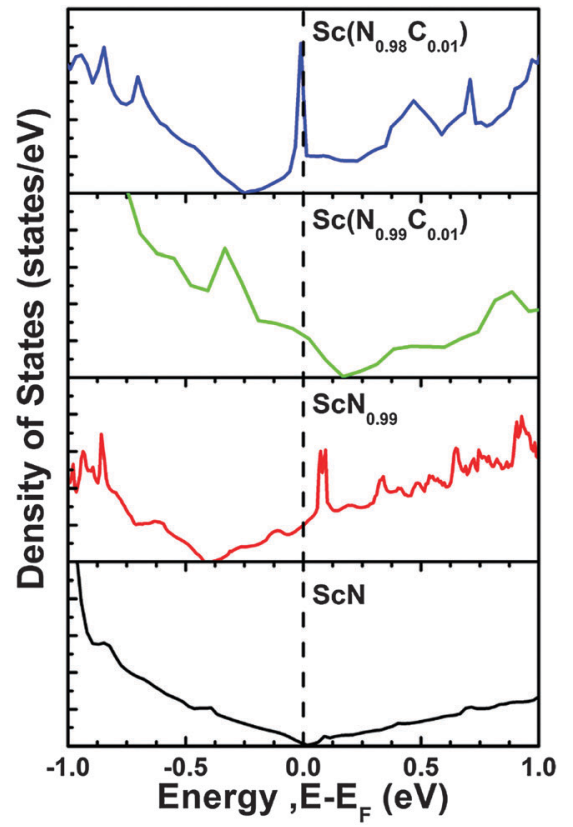

Fig. 3 Example of effects of vacancies and dopants on the band structure of ScN (adapted from ref. 46). Bottom: Pure, stoichiometric ScN (note the inaccurate bandgap determination with GGA). Second from bottom: $1 \% N$ vacancies. Second from top: $1 \% C$ dopants. Top: Both $C$ dopants and $\mathrm{N}$ vacancies.

ScN as a thermoelectric material; strategies for addressing this are discussed in Section $\mathrm{V}$ below. These tellurides are benchmark thermoelectric materials. Nonetheless, the scarcity ${ }^{45}$ of Te as well as legislative restrictions on the use of $\mathrm{Pb}$ limits their applicability outside niche applications. Hence, much effort is devoted to developing alternative materials. The early transition-metal nitrides are a class that was not much considered for this purpose until just a few years ago. From an application point-ofview, CrN-based materials are closer to application than $\mathrm{ScN}$-based ones, since the former are abundant, relatively inexpensive and can readily be made in large quantities by standard processing techniques both in thin films and bulk.

The unexpectedly high thermoelectric power factor of $\mathrm{ScN}$ can be explained based on band-structure features caused by impurities. A conceptual illustrative example is shown in Fig. 3 (adapted from ref. 46), where first-principles calculations show that the combination of (in this example) $\mathrm{C}$ dopants and $\mathrm{N}$ vacancies in ScN introduces a sharp variation in the density of states at the Fermi level. As described in Section II, to maximize $Z T$, the transport-distribution function should be a bounded delta function (for a given phonon $\kappa$ ), realized in practice as a large slope in the density of states near the Fermi level. Thus, the electronic structure of $\mathrm{ScN}$ - including vacancies and impurities $\mathrm{n}$ the level of $\sim 1$ at $\%$ - can mimic the ideal theoretical transport-distribution function, yielding a high power factor. ${ }^{46,47}$ The same conclusions are drawn from calculations with $\mathrm{O}$ or $\mathrm{F}$ dopants., ${ }^{3,46}$

\section{C. $\mathrm{CrN}$}

$\mathrm{CrN}$ is well known from applications as hard coatings, given its high hardness of $28 \mathrm{GPa}$ and good resistance to wear and corrosion. ${ }^{48-58}$
It can also be readily synthesized in bulk..$^{59,60}$ In addition to these properties, CrN exhibits a magnetic phase transition. ${ }^{19,61,62}$ Above the Néel temperature $\left(T_{\mathrm{N}}\right)$ of $286 \mathrm{~K}, \mathrm{CrN}$ is paramagnetic with cubic $(\mathrm{NaCl})$ crystal structure with a lattice parameter reported to range from 4.135 to $4.185 \AA^{63-66}$ Below this temperature, the structure is antiferromagnetic and orthorhombic.

The electrical properties of $\mathrm{CrN}$ can vary greatly; for example, various studies show resistivity values ranging from 1.7 to $350 \mathrm{~m} \Omega \mathrm{cm}^{4,55,60,67}$ As for the reported temperaturedependent behavior of the electrical resistivity around the cubic-to-orthorhombic phase transition, ${ }^{68}$ there is typically a jump in resistivity between these two semiconducting phases, though there are reports of metallic behavior for the orthorhombic phase. ${ }^{69}$ Part of this apparent discrepancy can be attributed to $\mathrm{CrN}$ being a narrow-bandgap semiconductor, where the presence of $\mathrm{N}$ vacancies may act as effective dopants yielding high electron concentrations and metallic-like behavior below the Néel temperature. ${ }^{70}$ In epitaxial thin films, it is possible to stabilize the cubic phase and suppress the phase transition to the orthorhombic phase ${ }^{71-73}$ In addition, Gall et al. ${ }^{72}$ suggested that the conducting behavior in CrN films is a hopping conduction mechanism, and the band gap of $\mathrm{CrN}$ depends on the correlation energy.

For thermoelectric properties, CrN exhibits high Seebeck coefficients of typically around $135 \mu \mathrm{V} \mathrm{K} \mathrm{K}^{-1}$ around room temperature and up to $200 \mu \mathrm{V} \mathrm{K}^{-1}$ at $600 \mathrm{~K}^{60}$ Also, its thermal conductivity is moderate at $\sim 1.7 \mathrm{~W} \mathrm{~m}^{-1} \mathrm{~K}^{-1}(\sim 1 / 5$ of that of $\mathrm{ScN}$ ). Nonetheless, the electrical resistivity is relatively high; in pure form, because the localized $3 \mathrm{~d}$ orbitals of Cr give large effective masses causing high Seebeck coefficients and resistivities. This was recently addressed by Quintela et $a l^{5}{ }^{5}$ who annealed as-deposited films in ammonia gas for $2 \mathrm{~h}$ at $800{ }^{\circ} \mathrm{C}$ to ensure that the films were fully stoichiometric and to improve the crystalline quality; yielding a large improvement in Seebeck coefficient and a hundredfold reduction in resistivity.

This further underscores that the early transition-metal nitrides hold unexpected promise as novel thermoelectric materials. However, in their pure form, neither $\mathrm{ScN}$ nor $\mathrm{CrN}$ are likely to reach all the way; experimental strategies and theoretically guided design approaches for reduction of the thermal conductivity with retained or increased power factors are needed.

\section{Theoretical methodology}

Electronic structure calculations based on density functional theory (DFT) $)^{74,75}$ and standard approximations for the exchangecorrelation energies like the local density approximation (LDA) and the different flavors of the generalized gradient approximation (GGA) ${ }^{76}$ are very efficient and most often accurate tools for phase stability and ground state related properties for most classes of materials. However, both the existing cases where this framework is insufficient in treatment of the quantum electronic problem, and perhaps more frequently, the neglect in theoretical studies of relevant vibrational, magnetic, and structural disorder, present in reality, is an obstacle in first-principles based 
calculations for real-world materials science problems. In fact, the early $3 \mathrm{~d}$ transition metal nitrides constitute an interesting illustration of several of these challenges. In $\mathrm{ScN}$, calculations of the bandgap using the Kohn-Sham orbital gap in GGA gives basically a zero-gap semiconductor, an underestimation effect well known and found for practically all semi-conductors. This is seen in the bottom curve in Fig. 2, from ref. 46. In VN, the rock-salt structure observed at room temperature and above is actually dynamically stabilized by anharmonic lattice vibrations and unstable at low temperature. ${ }^{77}$ This can lead to unphysical large atomic relaxation effects in VN-based systems if static, $0 \mathrm{~K}$, calculations are performed without symmetry constraints, e.g., with point defects or alloys. Finally, in $\mathrm{CrN}$, the effect of strong electron correlation is treatable on the level of LDA+U. ${ }^{70,78,79}$ However, most importantly the magnetic degree of freedom has caused considerable debate. In particular, the bulk modulus of paramagnetic rock-salt phase of $\mathrm{CrN}$ has been modeled using non-spin polarized calculations. ${ }^{80}$ However, the existence of local finite $\mathrm{Cr}$ moments, also above the magnetic ordering temperature, is crucial to consider in the theoretical modeling, ${ }^{81}$ otherwise the bulk modulus is greatly overestimated. This model, including local $\mathrm{Cr}$ moments in the paramagnetic phase, were later independently confirmed by new sets of experiments. ${ }^{82}$ The simultaneous presence of lattice vibrations and disordered magnetic moments in paramagnetic rock salt $\mathrm{CrN}$ causes further challenges for quantitative modeling. In fact, due to this complexity, the material has become a benchmark case for theoretical method development in the field. ${ }^{83-86}$ A similar finding of the importance of magnetism was also made for the bulk modulus of $\mathrm{Cr}_{2} \mathrm{AlC}$ and other Cr-containing so-called MAX phases. ${ }^{87}$ The crucial importance of the details of the nitrogen content and oxygen contamination levels pointed out in the previous section, also causes concern for the theoretical modeling of $\mathrm{CrN}$ as it couples with the vibrational and magnetic degrees of freedom. ${ }^{88}$

The current outstanding issue in this line of theoretical development, is associated with the unclear, material-specific, timescale for the propagation of the magnetic state as compared to the dynamics of the lattice. Recently, several methodological obstacles have been overcome, and methods for constrained local moments calculations ${ }^{89}$ and the derivation of Heisenberg-type exchange interactions, ${ }^{90}$ within the supercell based plane-wave electronic structure frameworks needed for $a b$ initio molecular dynamics now exist. The stage is set for a direct combinations of molecular and spin dynamics in an effective $a b$ initio manner with minimum or no free parameters. It would open up for first principles based calculations of lattice and magnetic thermal conductivity in the paramagnetic phase of magnetic materials, such as $\mathrm{CrN}$.

In the case of first-principles modeling of substitutionally disordered nitride alloys, e.g., $\mathrm{Cr}_{1-x} \mathrm{Al}_{x} \mathrm{~N}$ and $\mathrm{Ti}_{1-x} \mathrm{Al}_{x} \mathrm{~N},{ }^{91}$ the configurational problem arise as the crystallographic unit cell is no longer sufficient to describe the material. In a completely random alloy, the components are stochastically distributed on the lattice sites, metal sublattice in the case of a nitride alloy, implying lack of long range order and existence of many different local chemical environments of the atoms. The most reliable method to model such materials is the special quasirandom structure (SQS) method $^{92}$ introduced for transition metal nitride alloys in a study of $\mathrm{Ti}_{1-x} \mathrm{Al}_{x} \mathrm{~N} .{ }^{93}$ Using the SQS approach the mixing thermodynamics of the alloys can be directly modeled within a mean-field approximation for the configurational entropy. Also, e.g., the piezoelectric properties can be calculated directly. ${ }^{94,95}$ For the alloys of transition metal nitrides, and group-13 nitrides like AlN, such modeling has revealed important information about the mixing trends, i.e., if the supersaturated alloys obtained in the out-of-equilibrium synthesis at low temperature, will phase separate, order, or stay as a solid solutions when subject to the temperatures needed to induce metal-sublattice diffusion, e.g., in several $(\mathrm{Sc}, \mathrm{M}) \mathrm{N}^{96}$ and $(\mathrm{Cr}, \mathrm{M}) \mathrm{N}^{97}$ alloys.

It should be noted that real alloys under equilibrium conditions always display some degree of partial short-range ordering or, short-range clustering. This can of course also be the case for a metastable supersaturated solid solution grown with out-ofequilibrium techniques. However, in lack of a priori knowledge of such tendencies, the ideal random SQS approach is a welldefined, unbiased starting point for e.g. more intricate clusterexpansion approaches of the configurational thermodynamics. ${ }^{98}$ Outstanding issues here include the difficulty to include vibrational free energy, and in particular anharmonic contributions, into the configurational thermodynamics analysis in an accurate and efficient manner. ${ }^{99}$

With a reliable state-of-the art theoretical description of the materials equilibrium properties, the door opens for accurate calculations of properties. However, such calculations involves drastically different levels of complexity depending on which property that is needed. The properties needed for predicting the piezoelectric response of a material are second-order strainderivatives of ground state energies, elastic constants, and firstorder strain-derivatives of polarization. ${ }^{100}$ These are relatively straightforward to calculate accurately from first-principles with the complexities arising mostly from their tensorial nature, where care must be taken in the case of disordered alloys. ${ }^{101}$

The properties needed for understanding thermoelectric behavior of a material, on the other hand, are quite challenging to derive directly and accurately from first-principles, because the thermoelectric figure of merit includes both electronic and thermal transport and the entropy involves non-equilibrium transport processes. $A b$ initio calculation of thermoelectric parameters is addressed by Boltzmann transport theory, ${ }^{16,102}$ but involves an unknown scattering parameter, the relaxation time $\tau$. For the Seebeck coefficient (and Hall coefficient), $\tau$ cancels out if it is isotropic and constant with respect to energy. However, electrical and (electronic) thermal conductivities can only be determined either as a function of $\tau$ or by fitting to experimentally determined values ${ }^{103}$ of $\tau$, placing a substantial limitation on these computational approaches. Such calculations of the latter parameters are therefore not truly ab initio but restricted to materials for which experimental data of $\tau$ (or parameters from which $\tau$ can be calculated) are available. Ongoing method development is therefore devoted to finding methods for computing these from 
first principles. Examples are the recent work of Faghaninia et al. ${ }^{104}$ who derived an ab initio approach for computing these properties in the low-electric-field limit, and efforts to incorporate lowtemperature effect of phonon drag. ${ }^{105,106}$

\section{Ternary systems}

As proposed above, ScN and $\mathrm{CrN}$ are promising for thermoelectrics and based on cheap raw materials, while a reduced thermal conductivity of $\mathrm{ScN}$ or reduced electrical resistivity of $\mathrm{CrN}$ would be required for actual applications. This can be addressed in a $\mathrm{Cr}_{1-x} \mathrm{Sc}_{x} \mathrm{~N}$ solid solution, which is thermodynamically stable at high temperature in cubic NaCl-structured form. ${ }^{107}$ The fact that the $3 \mathrm{~d}$ orbitals in Sc are empty can be exploited as a means of delocalizing the electrons in $3 \mathrm{~d}$ orbitals, resulting in electrical conductivity reduction ${ }^{107}$ and possibly also thermal-conductivity reduction due to alloy scattering. We have recently shown that the Seebeck coefficient of Sc-rich $\mathrm{Cr}_{1-x} \mathrm{Sc}_{x} \mathrm{~N}$ solid solution epitaxial thin films does indeed increase compared to pure $\mathrm{ScN}$ and that the thermoelectric properties of $\mathrm{CrN}$ are largely retained in Cr-rich $\mathrm{Cr}_{1-x} \mathrm{Sc}_{x} \mathrm{~N}$ solid solutions. ${ }^{107}$

$\mathrm{ScN}$-based solid solutions are further important, because of the interest caused by the exceptionally high piezoelectric coupling coefficient in (Sc,Al)N alloys. ${ }^{1,108}(\mathrm{Sc}, \mathrm{Al}) \mathrm{N}$ and $(\mathrm{Sc}, \mathrm{Ga}) \mathrm{N}$ alloys were recently reviewed by Moram and $\mathrm{Zhang}^{109}$ and the reader is referred there. $(\mathrm{Sc}, \mathrm{Mn}) \mathrm{N}$ was investigated by Saha et al. ${ }^{110}$

Alloy scattering is one of the standard strategies for thermoelectric materials for reduction of the lattice thermal conductivity; other approaches are superlattices, nanoinclusions, or grain boundaries. ${ }^{6,111-114}$ Furthermore, the peaks in the density of states at the Fermi level causing high Seebeck coefficient is traditionally associated with reduced thermodynamic stability. ${ }^{115,116}$ For this reason, the search for optimal thermoelectric materials may be fruitful among metastable materials synthesized with far-from-equilibrium techniques, such as magnetron-sputtered metastable nitride thin film alloys, with the reservation that the metastable nature of such materials would place a limit on high-temperature long-term use.

$\mathrm{ScN}$ - and CrN-based systems are interesting model systems for these general research questions. ${ }^{96}$ In particular, Sc is naturally isotope-pure, thus lacking isotope reduction of thermal conductivity. Consequently, the possibilities to substantially reduce the thermal conductivity by alloying or nanostructural engineering are particularly promising in this material. If the thermal conductivity can be reduced, ScN-based materials could potentially be applied at elevated temperatures, where bulk diffusion can be activated and the thermodynamics of mixing between $\mathrm{ScN}$ and the alloying or superlattice component becomes relevant. Superlattices might intermix, alloys could order or phase-separate, and nanostructures might be dissolved in the matrix. All these processes will most likely affect thermoelectric properties.

Superlattices are of great interest for thermoelectrics, since they may allow for both the reduction of the lattice thermal conductivity and the quantum confinement of electrons. The first thermoelectric superlattice devices were made from combinations of the semiconductors $\mathrm{Bi}_{2} \mathrm{Te}_{3} / \mathrm{Sb}_{2} \mathrm{Te}_{3} \cdot{ }^{117} \mathrm{~A}$ different approach is to combine the high electron concentrations of ultrathin metallic layers (e.g., TiN or $\mathrm{ZrN}$ ) inserted between semiconductor barriers (e.g., $\mathrm{CrN}, \mathrm{ScN}$ ), the sharp asymmetry in the conduction electron distribution near the Fermi energy may be achieved for possible substantial improvements in $Z T .{ }^{118}$ Furthermore, it has been demonstrated that the high interface density in a superlattice can reduce the thermal conductivity in $\mathrm{ScN} /(\mathrm{Zr}, \mathrm{W}) \mathrm{N}$ superlattices. ${ }^{44}$ This is illustrated in Fig. 4, from Rawat et al. ${ }^{44}$

A theoretically guided approach to implementing these strategies are to use density functional theory calculations to investigate the effect of mixing thermodynamics in order to determine phase stability of ScN-based solid solutions of relevance for lattice thermal conductivity reduction. Our results demonstrated $^{96}$ that at $800{ }^{\circ} \mathrm{C}$ the free energy of mixing for $(\mathrm{Sc}, \mathrm{Y}) \mathrm{N},(\mathrm{Sc}, \mathrm{La}) \mathrm{N},(\mathrm{Sc}, \mathrm{Gd}) \mathrm{N}$, and $(\mathrm{Sc}, \mathrm{In}) \mathrm{N}$ exhibits a thermodynamic tendency for phase separation at high temperature. In addition, for the 50:50 $\mathrm{Sc}: \mathrm{M}(\mathrm{M}=\mathrm{V}, \mathrm{Nb}$, or Ta) ratio, the $(\mathrm{Sc}, \mathrm{V}) \mathrm{N},(\mathrm{Sc}, \mathrm{Nb}) \mathrm{N}$, and $(\mathrm{Sc}, \mathrm{Ta}) \mathrm{N}$ exhibit a stable ternary inherently nanolaminated phase ${ }^{119}$ with the $\mathrm{ScTaN}_{2}$-type structure. On the other hand, at $800{ }^{\circ} \mathrm{C}$, the (Sc,Ti)N, (Sc,Zr)N, (Sc,Hf)N, and $(\mathrm{Sc}, \mathrm{Lu}) \mathrm{N}$ are thermodynamically stable in disordered $\mathrm{B} 1(\mathrm{NaCl})$ solid solutions, rather than in the ordered solid solutions which are stable at $0 \mathrm{~K}$. This last point is shown in Fig. 5 (from ref. 96), which shows (Fig. 5(a)) a comparison of the calculated mixing enthalpies of substitutionally disordered solid solution, ordered solid solutions and $\mathrm{ScTaN}_{2}$-type structure phase of $(\mathrm{Sc}, \mathrm{M}) \mathrm{N}$, as a function of $\mathrm{MN}$ content where $\mathrm{M}=\mathrm{Ti}, \mathrm{Zr}$, and Hf, and (Fig. 5(b)) calculated equilibrium lattice parameter for the rocksalt (B1) solid solution as a function of MN content.

These results enabled us to suggest suitable materials for the different possible strategies for reduction of the lattice thermal conductivity of ScN. Since the heavy element Lu has a mixing tendency with $\mathrm{ScN}$ and has the same number of valence electrons, it is an appropriate choice for solid-solution reduction of the thermal conductivity. YN, LaN, GdN, AlN, GaN, InN, have a thermodynamic tendency for phase separation with $\mathrm{ScN}$ and thus constitute good alloying elements if decomposition to form nanoinclusions is the strategy. The three former can be

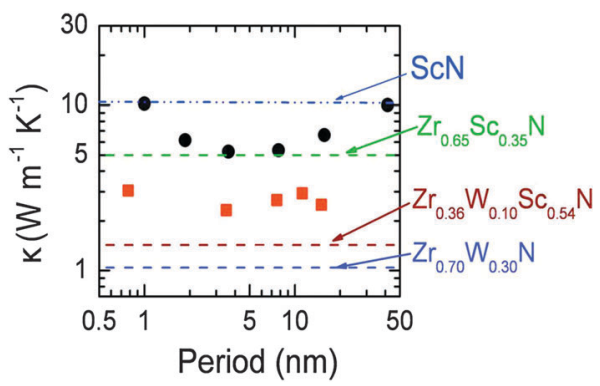

Fig. 4 Illustration of superlattice reduction in thermal conductivity, from Rawat et al. ${ }^{44}$ Cross-plane thermal conductivity of $300 \mathrm{~nm}$ thick $\mathrm{ZrN} / \mathrm{ScN}$ (dots) and $\mathrm{Zr}_{0.64} \mathrm{~W}_{0.36} \mathrm{~N} / \mathrm{ScN}$ (squares) multilayers. Superimposed on the plot are horizontal lines corresponding to the experimentally determined lattice component of thermal conductivity, i.e., the alloy limit of different alloys of $\mathrm{ZrN}, \mathrm{ScN}$, and $\mathrm{W}_{2} \mathrm{~N}$. (Copyright American Institute of Physics, used with permission). 
(a)

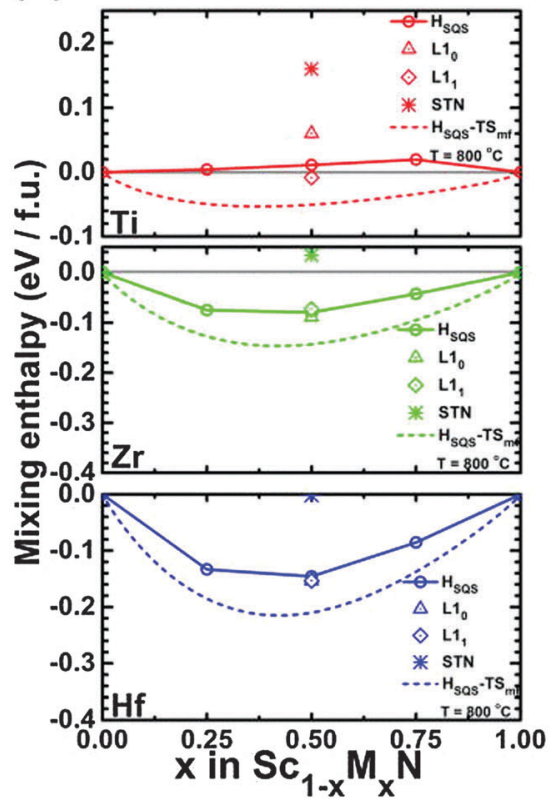

(b)

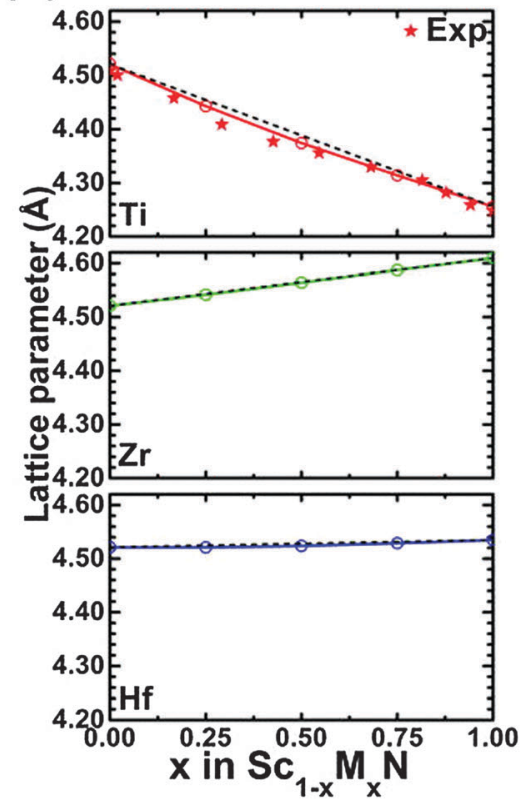

Fig. 5 (a) Comparison of the calculated mixing enthalpies of substitutionally disordered solid solution, ordered solid solutions and ScTaN ${ }_{2}$-type structure phase of $\mathrm{Sc}_{1-x} \mathrm{M}_{x} \mathrm{~N}$, as a function of $\mathrm{MN}$ content where $\mathrm{M}=\mathrm{Ti}, \mathrm{Zr}$, and $\mathrm{Hf}$, respectively. (b) Calculated equilibrium lattice parameter for rocksalt (B1) $\mathrm{Sc}_{1-x} \mathrm{M}_{x} \mathrm{~N}$ solid solution as a function of $\mathrm{MN}$ content where $\mathrm{M}=\mathrm{Ti}, \mathrm{Zr}$, and Hf, respectively. The black line indicates Vegard's rule. For (Sc,Ti) $\mathrm{N}$, experimental data from Gall et al. ${ }^{22}$ are shown with stars. From ref. 96 (Copyright American Institute of Physics, used with permission).

used for superlattices, since they in addition are isostructural with ScN. That, in combination with the thermodynamic tendency for phase separation, would render the superlattice structure stable. The wurtzite AlN, GaN, and InN are not suited for this purpose because of the difference in crystal structure, but their alloys with $\mathrm{ScN}$ or other $\mathrm{NaCl}$-structure nitrides can be, as described below. ${ }^{120-122}$ The mixing thermodynamics of these alloy systems can be understood by considering the effect of the factors of volume mismatch, favoring phase separation, and an electronic structure effect of delocalization of extra d-electrons to empty Sc $3 \mathrm{~d}-\mathrm{t}_{2 \mathrm{~g}}$ states, favoring mixing.

Important experimental demonstrations of these principles are the works of Saha et al. who stabilized cubic $(\mathrm{Sc}, \mathrm{Al}) \mathrm{N}$ latticematched $^{121}$ to TiN and (Ti,W)N in TiN/(Sc,Al)N and (Ti,W)N/ $(\mathrm{Sc}, \mathrm{Al}) \mathrm{N}$ superlattices exhibiting enhanced hardness ${ }^{122}$ and large reduction in thermal conductivity. ${ }^{123}$ Here, the combination of superlattice scattering and heavy-element alloying (with W) allowed for thermal conductivities down to $1.7 \mathrm{~W} \mathrm{~m}^{-1} \mathrm{~K}^{-1}$ (compared to $8-10 \mathrm{~W} \mathrm{~m}^{-1} \mathrm{~K}^{-1}$ for pure $\mathrm{ScN}$ ). Nonetheless, as discussed above, these superlattice structures are metastable and limited in use to the medium-temperature range. Around $800{ }^{\circ} \mathrm{C}$, where bulk diffusion becomes dominant, their long-term thermal stability would be compromised due to intermixing. This was demonstrated by Schreoder et al. who showed that TiN/(Sc,Al)N superlattices intermix heavily at elevated temperature. ${ }^{120}$

Finally, we note that recently theoretical calculations has been used to suggest further alternatives to the $\mathrm{ScN}$ and $\mathrm{CrN}$ based semiconducting systems. In particular, by combining group-4 transition metals with a group-2 alkaline-earth metal in equal amounts, novel semiconducting systems have been predicted, like $\mathrm{Ti}_{0.5} \mathrm{Mg}_{0.5} \mathrm{~N}^{124}$ and the wurtzite-structure $\left(\mathrm{TM}_{0.5}, \mathrm{M}_{0.5}\right)_{x} \mathrm{Al}_{1-x} \mathrm{~N}$ alloys are investigated for piezoelectric properties. ${ }^{125,126}$ These recent studies demonstrate the superior speed with which computationally based approaches can scan large pools of complex, uncharted materials and suggest candidates for a given property, before experimental verification is pursued. This emphasizes the promise for a future of theoretically driven materials discoveries, at least in the cases where the theoretical accuracy and methodological reliability is well established.

\section{Concluding remarks}

We have reviewed the present state of research on early transitionmetal nitrides, primarily based on $\mathrm{ScN}$ and $\mathrm{CrN}$, for thermoelectric energy harvesting. These materials also constitute well-defined model systems for theory-guided approaches investigating thermodynamics of mixing for alloying and nanostructural design for optimization of phase stability and band structure, in order to improve thermoelectric properties. This is most notable as thermoelectric properties per se - unlike piezoelectric properties - are challenging to reliably calculate by $a b$ initio methods. This can be used to guide the implementation of strategies for reduction of the lattice thermal conductivity; alloy scattering, superlattices, and nanoinclusions.

\section{Acknowledgements}

The authors acknowledge funding from the European Research Council under the European Community's Seventh Framework 
Programme (FP/2007-2013)/ERC grant agreement no. 335383, the Swedish Government Strategic Research Area in Materials Science on Functional Materials at Linköping University (Faculty Grant SFO-Mat-LiU No. 2009 00971), the Swedish Foundation for Strategic Research (SSF) through the Future Research Leaders 5 program, and the Swedish Research Council (VR) under project no. 621-2012-4430, project no. 621-2011-4417, and International Career Grant 330-2014-6336.

\section{References}

1 M. Akiyama, T. Kamohara, K. Kano, A. Teshigahara, Y. Takeuchi and N. Kawahara, Adv. Mater., 2009, 21, 593.

2 S. Kerdsongpanya, N. Van Nong, N. Pryds, A. Zukauskaite, J. Jensen, J. Birch, J. Lu, L. Hultman, G. Wingqvist and P. Eklund, Appl. Phys. Lett., 2011, 99, 232113.

3 P. V. Burmistrova, J. Maassen, T. Favaloro, B. Saha, S. Salamat, Y. R. Koh, M. S. Lundstrom, A. Shakouri and T. D. Sands, J. Appl. Phys., 2013, 113, 153704.

4 C. X. Quintela, B. Rodriguez-González and F. Rivadulla, Appl. Phys. Lett., 2014, 104, 022103.

5 C. X. Quintela, J. P. Podkaminer, M. N. Luckyanova, T. R. Paudel, E. L. Thies, D. A. Hillsberry, D. A. Tenne, E. Y. Tsymbal, G. Chen, C.-B. Eom and F. Rivadulla, Adv. Mater., 2015, 27, 3032.

6 G. J. Snyder and E. S. Toberer, Nat. Mater., 2008, 7, 105.

7 C. J. Vineis, A. Shakouri, A. Majumdar and M. G. Kanatzidis, Adv. Mater., 2010, 22, 3970.

8 J. R. Sootsman, D. Y. Chung and M. G. Kanatzidis, Angew. Chem., Int. Ed., 2009, 48, 8616.

9 L. D. Hicks and M. S. Dresselhaus, Phys. Rev. B: Condens. Matter Mater. Phys., 1993, 47, 12727.

10 R. Venkatasubramanian, E. Siivola, T. Colpitts and B. O'Quinn, Nature, 2001, 413, 597.

11 C. B. Vining, Nat. Mater., 2009, 8, 83.

12 H. Liu, X. Shi, F. Xu, L. Zhang, W. Zhang, L. Chen, Q. Li, C. Uher, T. Day and G. J. Snyder, Nat. Mater., 2012, 11, 422.

13 R. J. Mehta, Y. Zheng, C. Karthik, B. Singh, R. W. Siegel, T. Borca-Tasciuc and G. Ramanath, Nat. Mater., 2012, 11, 233.

14 C. J. Vineis, A. Shakouri, A. Majumdar and M. G. Kanatzidis, Adv. Mater., 2010, 22, 3970.

15 J. R. Sootsman, D. Y. Chung and M. G. Kanatzidis, Angew. Chem., Int. Ed., 2009, 48, 8616.

16 G. D. Mahan and J. O. Sofo, Proc. Natl. Acad. Sci. U. S. A., 1996, 93, 7436.

17 Z. Y. Fan, H. Q. Wang and J.-C. Zheng, J. Appl. Phys., 2011, 109, 073713.

18 R. S. Ningthoujam and N. S. Gajbhiye, Prog. Mater. Sci., 2015, 70, 50 .

19 L. M. Corliss, N. Elliott and J. M. Hastings, Phys. Rev., 1960, 117, 929.

20 A. Herwadkar and W. R. L. Lambrecht, Phys. Rev. B: Condens. Matter Mater. Phys., 2009, 79, 035125.

21 B. Alling, Phys. Rev. B: Condens. Matter Mater. Phys., 2010, 82, 054408.
22 D. Gall, I. Petrov and J. E. Greene, J. Appl. Phys., 2001, 89, 401.

23 L. E. Toth, Transition Metal Carbides and Nitrides, Academic Press, New York, 1971.

24 S. W. King, R. F. Davis and R. J. Nemanich, J. Vac. Sci. Technol., A, 2014, 32, 061504.

25 J. P. Dismukes, W. M. Yim, J. J. Tietjen and R. E. Novak, J. Cryst. Growth, 1971, 9, 295.

26 J. P. Dismukes, W. M. Yim, J. J. Tietjen and R. E. Novak, R.C.A. Review, 1970, 31, 680.

27 G. Travaglini, F. Marabelli, R. Monnier, E. Kaldis and P. Wachter, Phys. Rev. B: Condens. Matter Mater. Phys., 1986, 34, 3876.

28 W. R. L. Lambrecht, Phys. Rev. B: Condens. Matter Mater. Phys., 2000, 62, 13538.

29 C. Stampfl, W. Mannstadt, R. Asahi and A. J. Freeman, Phys. Rev. B: Condens. Matter Mater. Phys., 2001, 63, 155106.

30 D. Gall, M. Städele, K. Järrendahl, I. Petrov, P. Desjardins, R. T. Haasch, T. Y. Lee and J. E. Greene, Phys. Rev. B: Condens. Matter Mater. Phys., 2001, 63, 125119.

31 M. A. Moram, Z. H. Barber and C. J. Humphreys, Thin Solid Films, 2008, 516, 8569.

32 R. Deng, B. D. Ozsdolay, P. Y. Zheng, S. V. Khare and D. Gall, Phys. Rev. B: Condens. Matter Mater. Phys., 2015, 91, 045104.

33 D. Gall, I. Petrov, N. Hellgren, L. Hultman, J. E. Sundgren and J. E. Greene, J. Appl. Phys., 1998, 84, 6034.

34 D. Gall, I. Petrov, P. Desjardins and J. E. Greene, J. Appl. Phys., 1999, 86, 5524.

35 J. M. Gregoire, S. D. Kirby, G. E. Scopelianos, F. H. Lee and R. B. van Dover, J. Appl. Phys., 2008, 104, 074913.

36 Y. Oshima, G. E. Villora and K. Shimamura, J. Appl. Phys., 2014, 115, 153508.

37 H. A. Al-Brithen, A. R. Smith and D. Gall, Phys. Rev. B: Condens. Matter Mater. Phys., 2004, 70, 045303.

38 T. D. Moustakas, R. J. Molnar and J. P. Dismukes, Proc. - Electrochem. Soc., 1996, 96(11), 197.

39 H. A. H. Al-Brithen, E. M. Trifan, D. C. Ingram, A. R. Smith and D. Gall, J. Cryst. Growth, 2002, 242, 345.

$40 \mathrm{H}$. Al-Brithen and A. R. Smith, Appl. Phys. Lett., 2000, $77,2485$.

41 M. A. Moram, T. B. Joyce, P. R. Chalker, Z. H. Barber and C. J. Humphreys, Appl. Surf. Sci., 2006, 252, 8385.

42 M. A. Moram, S. V. Novikov, A. J. Kent, C. Norenberg, C. T. Foxon and C. J. Humphreys, J. Cryst. Growth, 2008, 310, 2746.

43 J. R. Sootsman, et al., Angew. Chem., Int. Ed., 2008, 47, 8618. 44 V. Rawat, Y. K. Koh, D. G. Cahill and T. D. Sands, J. Appl. Phys., 2009, 105, 024909.

45 R. Amatya and R. J. Ram, J. Electron. Mater., 2012, 41, 1011. 46 S. Kerdsongpanya, B. Alling and P. Eklund, Phys. Rev. B: Condens. Matter Mater. Phys., 2012, 86, 195140.

47 M. G. Moreno-Armenta and G. Soto, Comput. Mater. Sci., 2007, 40, 275.

48 H. C. Barshilia, N. Selvakumar, B. Deepthi and K. S. Rajam, Surf. Coat. Technol., 2006, 201, 2193. 
49 G. G. Fuentes, R. Rodriguez, J. C. Avelar-Batista, J. Housden, F. Montalá, L. J. Carreras, A. B. Cristóbal, J. J. Damborenea and T. J. Tate, J. Mater. Process. Technol., 2005, 167, 415.

50 M. L. Kuruppu, G. Negrea, I. P. Ivanov and S. L. Rohde, J. Vac. Sci. Technol., A, 1998, 16, 1949.

51 T. Polcar, T. Kubart, R. Novák, L. Kopecký and P. Široký, Surf. Coat. Technol., 2005, 193, 192.

52 T. Polcar, N. M. G. Parreira and R. Novák, Surf. Coat. Technol., 2007, 201, 5228.

53 J. Lin, W. D. Sproul and J. J. Moore, Mater. Lett., 2012, 89, 55.

54 J. Lin, W. D. Sproul and J. J. Moore, Surf. Coat. Technol., 2012, 206, 2474.

55 D. Gall, C.-S. Shin, T. Spila, M. Odén, M. J. H. Senna, J. E. Greene and I. Petrov, J. Appl. Phys., 2002, 91, 3589.

56 C. Petrogalli, L. Montesano, M. Gelfi, G. M. La Vecchia and L. Solazzi, Surf. Coat. Technol., 2014, 258, 878.

57 J. Lin, N. Zhang, W. D. Sproul and J. J. Moore, Surf. Coat. Technol., 2012, 206, 3283.

58 N. Beliardouh, K. Bouzid, C. Nouveau, B. Tlili and M. Walock, Tribol. Int., 2015, 82, 443.

59 P. S. Herle, M. Hegde, N. Vasathacharya, S. Philip, M. R. Rao and T. Sripathi, J. Solid State Chem., 1997, 134, 120.

60 O. Jankovský, D. Sedmidubský, Š. Huber, P. Šimek and Z. Sofer, J. Eur. Ceram. Soc., 2014, 34, 4131.

61 A. Filippetti, W. E. Pickett and B. M. Klein, Phys. Rev. B: Condens. Matter Mater. Phys., 1999, 59, 7043.

62 A. Filippetti and N. A. Hill, Phys. Rev. Lett., 2000, 85, 5166.

63 C. Constantin, M. B. Haider, D. Ingram and A. R. Smith, Appl. Phys. Lett., 2004, 85, 6371.

64 P. Hones, M. Diserens, R. Sanjinés and F. Lévy, J. Vac. Sci. Technol., B: Microelectron. Nanometer Struct.-Process., Meas., Phenom., 2000, 18, 2851.

65 K. Inumaru, K. Koyama, N. Imo-oka and S. Yamanaka, Phys. Rev. B: Condens. Matter Mater. Phys., 2007, 75, 054416.

66 X. Y. Zhang, J. S. Chawla, B. M. Howe and D. Gall, Phys. Rev. B: Condens. Matter Mater. Phys., 2011, 83, 165205.

67 C. X. Quintela, F. Rivadulla and J. Rivas, Appl. Phys. Lett., 2009, 94, 152103.

68 P. S. Herle, M. Hegde, N. Vasathacharya, S. Philip, M. R. Rao and T. Sripathi, J. Solid State Chem., 1997, 134, 120.

69 C. Constantin, M. B. Haider and D. Ingram andn A. R. Smith, Appl. Phys. Lett., 2004, 85, 6371.

70 A. Herwadkar and W. R. L. Lambrecht, Phys. Rev. B: Condens. Matter Mater. Phys., 2009, 79, 035125.

71 X. Y. Zhang, J. S. Chawla, R. P. Deng and D. Gall, Phys. Rev. B: Condens. Matter Mater. Phys., 2011, 84, 073101.

72 D. Gall, C.-S. Shin, R. T. Haasch, I. Petrov and J. E. Greene, J. Appl. Phys., 2002, 91, 5882.

73 K. Inumaru, K. Koyama, N. Imo-oka and S. Yamanaka, Phys. Rev. B: Condens. Matter Mater. Phys., 2007, 75, 054416.

74 P. Hohenberg and W. Kohn, Phys. Rev., 1964, 136, B864.

75 W. Kohn and L. J. Sham, Phys. Rev., 1965, 140, A1133.

76 J. P. Perdew, K. Burke and M. Ernzerhof, Phys. Rev. Lett., 1996, 77, 3865.

77 A. Mei, O. Hellman, N. Wireklint, C. M. Schlepütz, D. V. Sangiovanni, B. Alling, A. Rockett, L. Hultman, I. Petrov and J. E. Greene, Phys. Rev. B: Condens. Matter Mater. Phys., 2015, 91, 054101.

78 A. S. Botana, V. Tran, D. Pardo, D. Baldomir and P. Blaha, Phys. Rev. B: Condens. Matter Mater. Phys., 2012, 85, 235118.

79 B. Alling, T. Marten and I. A. Abrikosov, Phys. Rev. B: Condens. Matter Mater. Phys., 2010, 82, 184430.

80 F. Rivadulla, M. Banobre-Lopez, C. X. Quintela, A. Pineiro, V. Pardo, D. Baldomir, M. A. Lopez-Quintela, J. Rivas, C. A. Ramos, H. Salva, J.-S. Zhou and J. B. Goodenough, Nat. Mater., 2009, 8, 947.

81 B. Alling, T. Marten and I. A. Abrikosov, Nat. Mater., 2010, 9, 283.

82 S. Wang, X. Yu, J. Zhang, M. Chen, J. Zhu, L. Wang, D. He, Z. Lin, R. Zhang, K. Leinenweber and Y. Zhao, Phys. Rev. B: Condens. Matter Mater. Phys., 2012, 86, 064111.

83 P. Steneteg, B. Alling and I. A. Abrikosov, Phys. Rev. B: Condens. Matter Mater. Phys., 2012, 85, 144404.

84 B. Alling, L. Hultberg, L. Hultman and I. A. Abrikosov, Appl. Phys. Lett., 2013, 102, 031910.

85 N. Shulumba, B. Alling, O. Hellman, E. Mozafari, P. Steneteg, M. Odén and I. A. Abrikosov, Phys. Rev. B: Condens. Matter Mater. Phys., 2014, 89, 174108.

86 L. Zhou, F. Körmann, D. Holec, M. Bartosik, B. Grabowski, J. Neugebauer and P. H. Mayrhofer, Phys. Rev. B: Condens. Matter Mater. Phys., 2014, 90, 184102.

87 M. Dahlqvist, B. Alling and J. Rosén, J. Appl. Phys., 2013, 113, 216103.

88 E. Mozafari, B. Alling, P. Steneteg and I. A. Abrikosov, Phys. Rev. B: Condens. Matter Mater. Phys., 2015, 91, 094101.

89 P.-W. Ma and S. L. Dudarev, Phys. Rev. B: Condens. Matter Mater. Phys., 2015, 91, 054420.

90 A. Lindmaa, R. Lizarraga, E. Holmström, I. A. Abrikosov and B. Alling, Phys. Rev. B: Condens. Matter Mater. Phys., 2013, 88, 054414.

91 B. Alling, T. Marten, I. A. Abrikosov and A. Karimi, J. Appl. Phys., 2007, 102, 044314.

92 A. Zunger, S.-H. Wei, L. G. Ferreira and J. E. Bernard, Phys. Rev. Lett., 1990, 65, 353.

93 B. Alling, A. V. Ruban, A. Karimi, O. E. Peil, S. I. Simak, L. Hultman and I. A. Abrikosov, Phys. Rev. B: Condens. Matter Mater. Phys., 2007, 75, 045123.

94 F. Tasnádi, I. A. Abrikosov and I. Katardjiev, Appl. Phys. Lett., 2009, 94, 151911.

95 F. Tasnádi, B. Alling, C. Höglund, G. Wingqvist, J. Birch, L. Hultman and I. A. Abrikosov, Phys. Rev. Lett., 2010, 104, 137601.

96 S. Kerdsongpanya, B. Alling and P. Eklund, J. Appl. Phys., 2013, 114, 073512.

97 L. Zhou, D. Holec and P. H. Mayrhofer, J. Phys. D: Appl. Phys., 2013, 46, 365301.

98 B. Alling, A. V. Ruban, A. Karimi, L. Hultman and I. A. Abrikosov, Phys. Rev. B: Condens. Matter Mater. Phys., 2011, 83, 104203.

99 N. Shulumba, O. Hellman, Z. Raza, J. Barriero, B. Alling, F. Mücklich, I. A. Abrikosov and M. Odén, 2015, arXiv:1503.02459. 
100 F. Bernandini, V. Fiorentini and D. Vanderbilt, Phys. Rev. B: Condens. Matter Mater. Phys., 1997, 56, R10024.

101 F. Tasnádi, M. Odén and I. A. Abrikosov, Phys. Rev. B: Condens. Matter Mater. Phys., 2012, 85, 144112.

102 G. K. H. Madsen and D. J. Singh, Comput. Phys. Commun., 2006, 175, 67.

103 K. Kutorasiński, J. Tobola and S. Kaprzyk, Phys. Rev. B: Condens. Matter Mater. Phys., 2013, 87, 195205.

104 A. Faghaninia, J. W. Ager III and C. S. Lo, Phys. Rev. B: Condens. Matter Mater. Phys., 2015, 91, 235123.

105 B. Qiu, Z. Tian, A. Vallabhaneni, B. Liao, J. M. Mendoza, O. D. Restrepo, X. Ruan and G. Chen, EPL, 2105, 109, 57006.

106 J. Zhou, B. Liao, B. Qiu, S. Huberman, K. Esfarjani, M. S. Dresselhaus and G. Chen, Proc. Natl. Acad. Sci. U. S. A., 2015, 112, 14777.

107 S. Kerdsongpanya, $\mathrm{PhD}$ thesis, Linköping studies in science and technology, 2015, ISBN 978-91-7519-067-9.

108 M. A Caro, S. Zhang, T. Riekkinen, M. Ylilammi, M. A Moram, O. Lopez-Acevedo, J. Molarius and T. Laurila, J. Phys.: Condens. Matter, 2015, 27, 245901.

109 M. A. Moram and S. Zhang, J. Mater. Chem. A, 2014, 2, 6042 .

110 B. Saha, G. Naik, V. P. Drachev, A. Boltassseva, E. E. Marinero and T. D. Sands, J. Appl. Phys., 2013, 114, 063519.

111 C. J. Vineis, A. Shakouri, A. Majumdar and M. G. Kanatzidis, Adv. Mater., 2010, 22, 3970.

112 A. Shakouri, Annu. Rev. Mater. Res., 2011, 41, 399.

113 E. S. Toberer, L. L. Baranowski and C. Dames, Annu. Rev. Mater. Res., 2012, 42, 179.
114 K. Biswas, J. He, Q. Zhang, G. Wang, C. Uher, V. P. Dravid and M. G. Kanatzidis, Nat. Chem., 2011, 3, 160.

115 P. Eklund, M. Beckers, U. Jansson, H. Högberg and L. Hultman, Thin Solid Films, 2010, 518, 1851.

116 G. Hug, Phys. Rev. B: Condens. Matter Mater. Phys., 2006, 74, 184113.

117 R. Venkatasubramanian, E. Siivola, T. Colpitts and B. O’Quinn, Nature, 2001, 413, 597.

118 M. Zebarjadi, Z. Bian, R. Singh, A. Shakouri, R. Wortman, V. Rawat and T. D. Sands, J. Electron. Mater., 2009, 38, 960.

119 R. Niewa, D. A. Zherebtsov, W. Schnelle and F. R. Wagner, Inorg. Chem., 2004, 43, 6188.

120 J. L. Schroeder, B. Saha, M. Garbrecht, N. Schell, T. D. Sands and J. Birch, J. Mater. Sci., 2015, 50, 3200.

121 B. Saha, S. Saber, G. V. Naik, A. Boltasseva, E. A. Stach, E. P. Kvam and T. D. Sands, Phys. Status Solidi B, 2015, 252, 251.

122 B. Saha, S. K. Lawrence, J. L. Schroeder, J. Birch, D. F. Bahr and T. D. Sands, Appl. Phys. Lett., 2014, 105, 151904.

123 B. Saha, Y. R. Koh, J. Comparan, S. Sadasivam, J. L. Schroeder, M. Garbrecht, A. Mohammed, J. Birch, T. Fisher, A. Shakouri and T. D. Sands, Phys. Rev. B: Condens. Matter Mater. Phys., 2016, 93, 045311.

124 B. Alling, Phys. Rev. B: Condens. Matter Mater. Phys., 2014, 89, 085112.

125 C. Tholander, F. Tasnadi, I. A. Abrikosov, L. Hultman, J. Birch and B. Alling, Phys. Rev. B: Condens. Matter Mater. Phys., 2015, 92, 174119.

126 Y. Iwazaki, T. Yokoyama, T. Nishihara and M. Ueda, Appl. Phys. Express, 2015, 8, 061501. 